\title{
Pesquisa odontológica brasileira em regeneração óssea guiada: um estudo bibliométrico de quatro décadas
}

\author{
Brazilian dental research in guided bone regeneration: a bibliometric study of four decades \\ Investigación dental brasileña em regeneración ósea guiada: un estudio bibliométrico de cuatro \\ decadas
}

Igor Iuco Castro-Silva

ORCID: https://orcid.org/0000-0003-4815-6357 Universidade Federal do Ceará, Brasil

E-mail: igor.iuco@sobral.ufc.br

Lana Karine Araújo

ORCID: https://orcid.org/0000-0003-2200-8313 Universidade Federal do Ceará, Brasil

E-mail: lanakarine1380@gmail.com

Francisco Fábio Pereira de Souza

ORCID: https://orcid.org/0000-0002-3086-7954

Universidade Federal do Ceará, Brasil

E-mail: fabiosouzawin@gmail.com

José Sandro Ponte

ORCID: https://orcid.org/0000-0002-8111-4709

Universidade Federal do Ceará, Brasil

E-mail: drsandroponte@hotmail.com

Efigênia Maria de Sousa

ORCID: https://orcid.org/0000-0003-0191-3998 Universidade Federal do Ceará, Brasil E-mail: effipu@gmail.com

Haydee Gina Quispe Jiménez

ORCID: https://orcid.org/0000-0002-9622-410X Universidade Federal do Ceará, Brasil

E-mail: haydee_qj@hotmail.com

Francisco Valdicélio Ferreira

ORCID: https://orcid.org/0000-0002-6347-2844 Universidade Federal do Ceará, Brasil

E-mail: celionutri@gmail.com

Lara Gomes de Alcântara

ORCID: https://orcid.org/0000-0002-6037-4042 Universidade Federal do Ceará, Brasil

E-mail: laragomesalc@gmail.com

Maryane Breckenfeld Silva Diniz ORCID: https://orcid.org/0000-0002-2771-077X Universidade Federal do Ceará, Brasil E-mail: marybreckenfeld@gmail.com Yasmin Alves Teles Menezes

ORCID: https://orcid.org/0000-0002-1097-6977 Universidade Federal do Ceará, Brasil E-mail: yasmin.teles56@gmail.com

Carolina de Holanda Azevedo

ORCID: https://orcid.org/0000-0003-3107-9615 Universidade Federal do Ceará, Brasil E-mail: carolholanda33@gmail.com

Jacques Antonio Cavalcante Macie ORCID: https://orcid.org/0000-0002-2293-8433 Universidade Federal do Ceará, Brasil E-mail: jacques.maciel@ sobral.ufc.br

Marcelo Miranda de Melo

ORCID: https://orcid.org/0000-0003-2605-1319 Universidade Federal do Ceará, Brasil

E-mail: marcelomirandamelo3m@gmail.com 


\begin{abstract}
Resumo
Indicadores cienciométricos são úteis para dimensionar a produtividade acadêmica e seu impacto temporo-espacial. O objetivo deste estudo foi realizar um levantamento retrospectivo sobre pesquisas odontológicas brasileiras com biomateriais para regeneração óssea guiada. Resumos temáticos originais foram recuperados nos anais da SBPqO das últimas quatro décadas, perfazendo uma amostra de 1310 trabalhos, distribuídos nas categorias de aplicação, origem e composição do biomaterial, tipo de estudo, regionalidade e financiamento. A quantidade de pesquisas cresceu significativamente a partir da década de 1990 e se manteve estável até a década de 2010. Foi evidente a prevalência de pesquisa in vivo sobre clínica, estrutural e in vitro e a diminuição de outras formas de aplicação alternada com o incremento de enxerto ósseo, membrana e hemoderivado. A origem autógena foi a maior na década de 1980, xenógena nas décadas de 1990 e 2000 e aloplástica na década de 2010. Embora compósitos fossem mais prevalentes ao longo de todo o período estudado, a partir da década de 1990 polímeros foram mais frequentes do que cerâmicas e biovidros. A região Sudeste liderou pesquisas e fomentos ao longo de todas as décadas analisadas, apesar das regiões Sul, Nordeste e Centro-Oeste crescerem significativamente na temática e a região Norte não exibir produção. O estudo do escopo de biomateriais para a regeneração óssea guiada indica sua gradativa evolução técnico-científica e reforça sua relevância histórica para a pesquisa odontológica brasileira.
\end{abstract}

Palavras-chave: Odontologia; Regeneração óssea; Bibliometria; Substitutos ósseos; Materiais biocompatíveis.

\begin{abstract}
Scientometric indicators are useful for measuring academic productivity and its temporo-spatial impact. The aim of this study was to carry out a retrospective survey on Brazilian dental research with biomaterials for guided bone regeneration. Original thematic abstracts were retrieved from the SBPqO annals of the last four decades, making a sample of 1310 works, distributed in the categories of application, origin and composition of the biomaterial, type of study, regionality and financing. The amount of research has grown significantly from the 1990s and remained stable until the 2010s. The prevalence of in vivo research compared to clinical, structural, and in vitro studies was evident and the decrease other forms of application alternated with the increase in bone graft, membrane, and blood derivative. The autogenous origin was the largest in the 1980s, xenogeneic in the 1990s and 2000s and alloplastic in the 2010s. Although composites were more prevalent throughout the study period, from the 1990s polymers were more frequent than ceramics and bio-glass. The Southeast region has led research and financing over all the analyzed decades, even though the South, Northeast and Center-West regions have grown significantly in the theme and the North region has no production. The scoping study of biomaterials for guided bone regeneration indicates its gradual technical-scientific evolution and reinforces its historical relevance for Brazilian dental research.
\end{abstract}

Keywords: Dentistry; Bone regeneration; Bibliometrics; Bone substitutes; Biocompatible materials.

\title{
Resumen
}

Los indicadores cienciométricos son útiles para medir la productividad académica y su impacto temporal-espacial. El objetivo de este estudio fue realizar una investigacione retrospectiva sobre investigaciones odontológicas brasileñas con biomateriales para la regeneración ósea guiada. Se recuperaron resúmenes temáticos originales de los anales de la SBPqO de las últimas cuatro décadas, haciendo una muestra de 1310 trabajos, distribuidos en las categorías de aplicación, origen y composición del biomaterial, tipo de estudio, regionalidad y financiamiento. La cantidad de investigación ha crecido significativamente desde la década de 1990 y se mantuvo estable hasta la década de 2010. Se evidenció la prevalencia de la investigación in vivo frente a los estúdios clínicos, estructurales e in vitro y la disminución de otras formas de aplicación alternó con el aumento de injerto óseo, membranas y hemoderivados. El origen autógeno fue el mayor en la década de 1980, xenógeno en las décadas de 1990 y 2000 y aloplástico en la década de 2010. Aunque los composites fueron más predominantes durante el período de estudio, a partir de la década de 1990 los polímeros fueron más frecuentes que la cerámica y el biovidrio. La región Sudeste ha liderado la investigación y el financiamiento durante todas las décadas analizadas, a pesar de que las regiones Sur, Nordeste y Centro Oeste han crecido significativamente en el tema y la región Norte no tiene producción. El estudio de alcance de los biomateriales para la regeneración ósea guiada indica su paulatina evolución técnico-científica y refuerza su relevancia histórica para la investigación dental brasileña.

Palabras clave: Odontología; Regeneración ósea; Bibliometría; Sustitutos de huesos; Materiales biocompatibles.

\section{Introdução}

A gestão do saber é importante para a conversão do conhecimento empírico ou tácito em explícito e métodos apropriados de registro e divulgação são fundamentais para seu êxito (Câmara \& Nunes, 2021). Entradas et al. (2020) em estudo transnacional verificaram que o Brasil frente aos demais países no mundo continua a veicular suas pesquisas por meio de eventos e mídia científica tradicional, mas tem ultimamente experienciado redes sociais para agilizar a comunicação e gerar maior visibilidade para o público geral. A pesquisa acadêmica sempre está em movimento e a coleta de informação a partir de 
investigações prévias, sumarizadas em indicadores cienciométricos, pode ser uma rota útil para dimensionar determinada área de interesse ou redes colaborativas (Castro-Silva et al., 2021; Medeiros \& Vasconcelos, 2020).

A publicação científica favorece a comunicação entre estudantes e profissionais, na aquisição de competências ou atualização, moldando e dando existência à Odontologia nacional (Amorim et al., 2007). Sígolo et al. (2011) verificaram que o comportamento informacional da Odontologia no Brasil tem se modificado, para uma práxis mais dinâmica e acessível, diante da acelerada produção de novos conhecimentos. Amorim et al. (2007), analisando três periódicos odontológicos nacionais qualis B num recorte temporal de 15 anos, confirmaram a grande diversidade de temáticas existentes, com a categoria de materiais dentários ocupando o primeiro lugar e prevalecendo sobre demais disciplinas de cunho profissionalizante ou biológico. O destaque de materiais dentários também foi amplamente observado em estudos bibliográficos com resumos científicos de origem brasileira nas últimas décadas (Morosini et al., 2012; Dutra et al., 2011; Scariot et al., 2011; Aquino et al., 2009; Leles et al., 2006; Cavalcanti et al., 2004). Nobrega et al. (2020) afirmaram que a implantação das Diretrizes Curriculares Nacionais nos cursos de graduação em Odontologia em 2002 pode ter influenciado na produtividade da pesquisa nacional da década seguinte, com redução da dominância da região Sudeste, embora tenha se mantido o perfil tecnicista dos trabalhos, com predomínio das Ciências Odontológicas.

Relatos de pesquisas na forma de resumos em reuniões científicas profissionais compõem uma parte importante no processo de intercâmbio de informações científicas, oportunizando divulgação prévia de resultados de estudos finalizados e fortificação de linhas de pesquisa (Leles et al., 2006). A reunião anual da Sociedade Brasileira de Pesquisa Odontológica (SBPqO) se constitui no principal evento de caráter científico nacional, com alcance internacional e representa a Divisão Brasileira da International Association for Dental Research (IADR), onde milhares de resumos em nível de iniciação científica, mestrado e doutorado são apresentados por membros iniciantes, aspirantes ou efetivos (Aquino et al., 2009; Dias et al., 2008; Cavalcanti et al., 2004).

A bibliometria é uma técnica quantitativa com a finalidade de medir os índices de produção escrita e disseminação do conhecimento científico em tempo e espaço (Quevedo-Silva et al., 2016). Trabalhos científicos da SBPqO já subsidiaram análises bibliométricas específicas em diferentes temáticas, com maior frequência em odontopediatria (Morosini et al., 2012; Sampaio et al., 2006), patologia oral (Lúcio et al., 2012; Pereira et al., 2012) e endodontia (Gabardo et al., 2019; Pontes et al., 2017) e relatos isolados sobre imaginologia oral (Bezerra et al., 2011), pacientes com necessidades especiais (AlmeidaMarques et al., 2012), fitoterápicos (Barbosa et al., 2012), bioética (Marin et al., 2016), acupuntura (Fernandes Neto et al., 2019), má oclusão (Silva \& Padilha, 2018), terapia fotodinâmica em cariologia (Palmeira et al., 2019), odontologia forense (Perini et al., 2019) e microcefalia (Queiroz \& Soares, 2019).

A regeneração óssea guiada (ROG) em Odontologia é tema de pesquisas multidisciplinares e translacionais, por envolver desde o desenvolvimento laboratorial de biomateriais até as caracterizações biológicas normalizadas de validação para uso clínico (Bittencourt et al., 2014; Lomelino et al., 2011). Os biomateriais para ROG podem ser diferenciados quanto a aplicação (em especial, membranas para barreira e enxertos ósseos), origem (autógena, alógena, xenógena ou aloplástica) e composição (biocerâmicas e biovidros, polímeros ou compósitos), estando presentes na prática clínica e exibindo boa aceitação geral da classe odontológica (Araújo et al., 2020; Castro-Silva \& Coutinho, 2012). O Brasil exibe uma grande demanda multifatorial de perdas ósseas orofaciais (Castro-Silva et al., 2013). Entretanto, o uso de biomateriais para ROG na saúde pública por meio da inclusão nos procedimentos do Sistema Único de Saúde ainda é incipiente (Calasans-Maia et al., 2017).

Diante da ausência de trabalhos avaliando o impacto deste escopo nos resumos da SBPqO e para suprir tal lacuna de conhecimento, o objetivo deste estudo foi traçar por meio da análise bibliométrica um panorama da pesquisa odontológica brasileira sobre ROG nas últimas quatro décadas. 


\section{Metodologia}

Realizou-se um estudo quantitativo, bibliométrico, transversal, de análise documental online, delineado como uma revisão de escopo (Pereira et al., 2018). O estudo de escopo (scoping study ou scoping review) tem como objetivos mapear os principais conceitos que apoiam determinada área de conhecimento, examinar a extensão, alcance e natureza da investigação, sumarizar e divulgar os dados da investigação e identificar as lacunas de pesquisas existentes (Menezes et al., 2015).

Para a construção das estratégias de busca foi utilizada uma adaptação da estratégia PICO (P: Population, I: Intervention, C: Comparation e O: Outcome) que orienta a elaboração da pergunta de pesquisa exploratória e da busca bibliográfica e permite que o pesquisador localize, de maneira acurada, a melhor informação disponível (Menezes et al., 2015). A PVO é um acrônimo proposto por duas pesquisadoras brasileiras que constitui alternativa de revisões sistemáticas que fogem do caráter clínico da PICO, onde: "P” é a descrição do problema (população, contexto ou doença), "V” é a definição de limites ou variáveis e "O” é a definição dos desfechos (outcomes), desejados ou não (Menezes \& Novaes, 2018). Considerando este referencial, a busca na literatura dos artigos adotou para "P" o contexto de biomateriais para regeneração óssea guiada, "V" o limite temporal de quatro décadas e "O” as variáveis categóricas, de aplicação, origem e composição do biomaterial, tipo de estudo, regionalidade e financiamento.

A coleta dos dados brutos acessou os resumos publicados nos anais eletrônicos das reuniões anuais da SBPqO de 36 edições realizadas no período entre 1984 a 2019, disponíveis no site da entidade (SBPqO, 2020). Foi realizada leitura na íntegra de todos os resumos referenciados com calibração interna da equipe de pesquisa quanto à estratégia PVO. Como critérios de exclusão, foram removidas as revisões de literatura, trabalhos relacionados à implantes metálicos fixados após ROG e de instituições não brasileiras. Como critérios de inclusão, foram consideradas pesquisas originais com títulos ou conteúdos relacionados aos biomateriais aplicados em ROG e publicados na língua portuguesa ou inglesa.

Em um delineamento preliminar da amostragem representativa para este estudo de escopo, foi considerado um total inicial de 50.895 publicações, junto a nível de confiança de 95\%, margem de erro de 3\%, percentual correspondente à pesquisa de 50\% e percentual não correspondente à pesquisa de 50\%, para chegar à amostra estatística aproximada de 1.046, conforme o cálculo a seguir: $\eta=\left[50895(0,50)(0,50)\left(1,96^{2}\right)\right] /\left[(0,50)(0,50)\left(1,96^{2}\right)-50894\left(0,03^{2}\right)\right]=1.045,21$.

Para análise dos resultados, os resumos incluídos foram estratificados em quatro décadas consolidadas: 1980 (1984 a 1989), 1990 (1990 a 1999), 2000 (2000 a 2009) e 2010 (2010 a 2019). Para cada década, os dados absolutos e relativos das pesquisas foram classificados conforme as seguintes categorias: tipo de estudo, aplicação, origem e composição do biomaterial, região brasileira e financiamento da pesquisa. O tipo de estudo considerou um modelo: estrutural (teste físico, químico, mecânico e/ou condição extracorpórea simulada), in vitro (em cultura de células), in vivo (em animais não humanos, como rato, cão, camundongo, ovelha e macaco) e/ou clínico (em seres humanos). A aplicação incluiu: membrana para barreira (absorvível ou reabsorvível), enxerto ósseo, hemoderivado (PRP, PRF, i-PRF, L-PRF) e/ou outra forma de preenchimento de loja óssea (esponja hemostática, arcabouços gelatinosos de células ou fator de crescimento, proteínas de esmalte, cimento, cartilagem ou tecido adiposo). A origem envolveu: autógena (mesmo doador e receptor), alógena (doador e hospedeiro de mesma espécie), xenógena (doador e hospedeiro de espécies diferentes) e/ou aloplástica (sintética). A composição do biomaterial classificou como: cerâmica (produtos mineralizados, como hidroxiapatita e $\beta$-tricálcio fosfato); biovidros (vidro bioativo), polímero (produtos orgânicos, como colágeno, ácido polilático, politetrafluoretileno, policaprolactone ou similares) e/ou compósito (mistura de diferentes classes de biomateriais, como hidroxiapatita e colágeno). As variáveis divididas por décadas foram comparadas intergrupos, para avaliar a evolução temporo-espacial de cada categoria da produção científica temática.

Esta pesquisa foi realizada de acordo com os princípios bioéticos vigentes no Brasil para pesquisas considerando o uso de dados secundários de acesso público, em conformidade à Resolução do Conselho Nacional de Saúde nº $510 / 2016$. 


\section{Resultados}

A amostra final alcançou 1.310 resumos ou 2,57\% do total. Esse valor superou a amostra mínima prevista, reforçando sua significância dentro dos parâmetros estatísticos e poder de extrapolação para o conjunto geral de publicações analisadas. Foi possível constatar que a quantidade de pesquisas temáticas cresceu significativamente a partir da década de 1990, mais que o quíntuplo do observado em 1980, e se manteve estável até a década de 2010, conforme a Figura 1.

Figura 1 - Fluxograma da seleção de artigos incluídos no escopo de biomateriais para ROG nas últimas quatro décadas.
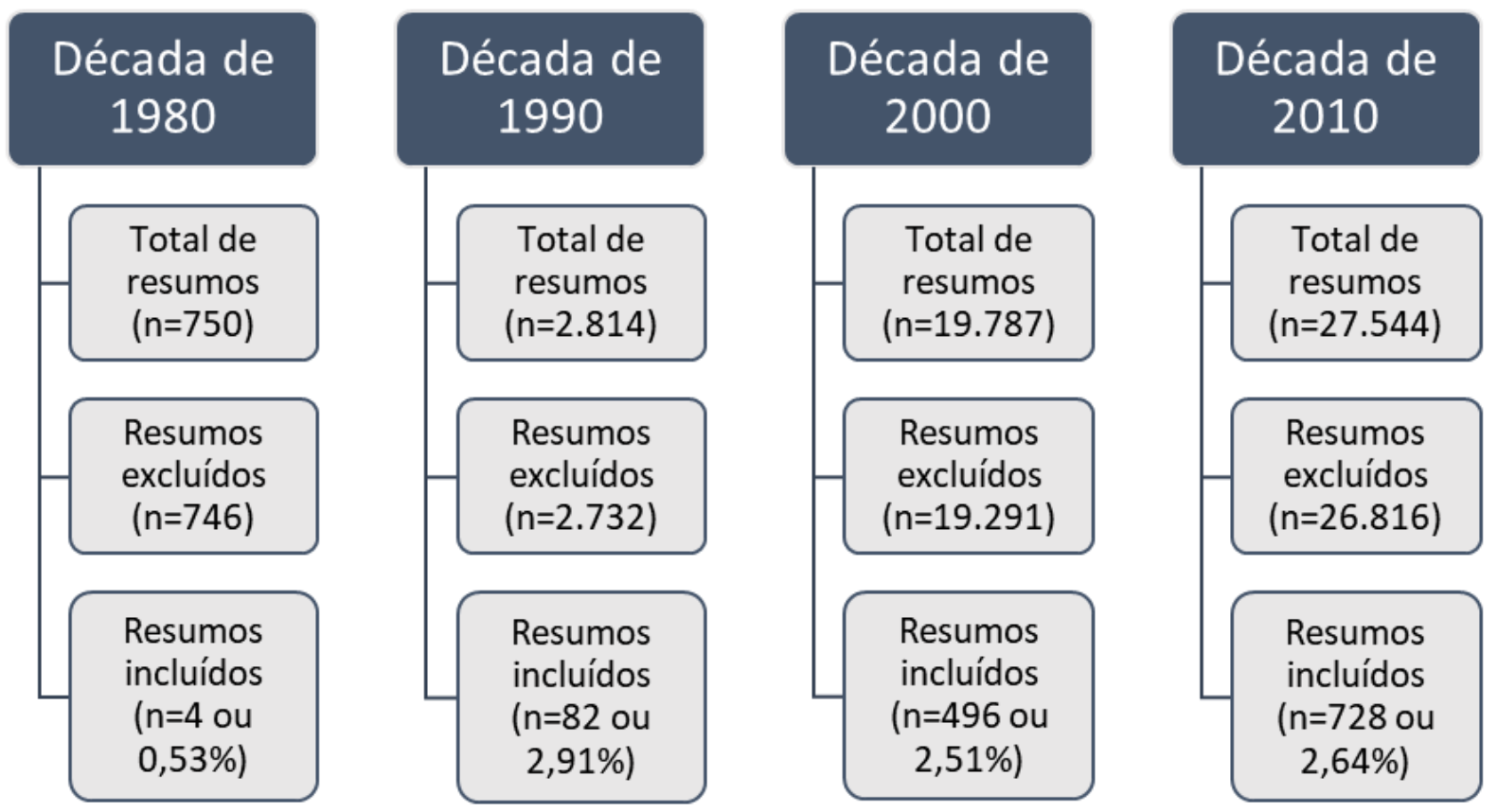

Fonte: Elaborada pelos autores, com base em dados de SBPqO (2020).

A Tabela 2 evidenciou um crescimento constante ao longo das décadas de tipo de estudo, com pesquisa in vivo mais prevalente que clínica, estrutural e in vitro, e aplicação, com outra forma de preenchimento diminuindo sua dominância e cedendo espaço para o incremento de pesquisas sobre enxerto ósseo, membrana e hemoderivado. Já origem e composição exibiram diferenças temporais específicas. Houve maior prevalência autógena na década de 1980, xenógena nas décadas de 1990 e 2000 e aloplástica na década de 2010. Embora compósitos estivessem mais prevalentes ao longo de todo o período estudado, a partir da década de 1990 notou-se que polímeros foram significativamente mais frequentes do que cerâmicas e biovidros. 
Tabela 2 - Frequência de pesquisas sobre biomateriais para ROG nas últimas quatro décadas conforme estudo, aplicação, origem e composição.

\begin{tabular}{|c|c|c|c|c|c|}
\hline \multicolumn{2}{|c|}{ Categoria } & \multicolumn{4}{|c|}{ Década } \\
\hline & & 1980 & 1990 & 2000 & 2010 \\
\hline \multirow{4}{*}{ Estudo* } & Estrutural & $0(0 \%)$ & $4(4,87 \%)$ & $25(5,05 \%)$ & $98(14,67 \%)$ \\
\hline & In vitro & $0(0 \%)$ & $3(3,65 \%)$ & $29(5,85 \%)$ & $91(13,62 \%)$ \\
\hline & In vivo & $3(75 \%)$ & $56(68,29 \%)$ & $416(84,04 \%)$ & $409(61,22 \%)$ \\
\hline & Clínico & $1(25 \%)$ & $24(29,26 \%)$ & $90(18,18 \%)$ & $137(20,50 \%)$ \\
\hline \multirow{4}{*}{ Aplicação* } & Membrana & $0(0 \%)$ & $33(40,24 \%)$ & $92(18,58 \%)$ & $144(21,55 \%)$ \\
\hline & Enxerto ósseo & $0(0 \%)$ & $31(37,80 \%)$ & $342(69,09 \%)$ & $300(44,91 \%)$ \\
\hline & Hemoderivado & $0(0 \%)$ & $3(3,65 \%)$ & $68(13,73 \%)$ & $80(11,97 \%)$ \\
\hline & Outra & $4(100 \%)$ & $54(65,85 \%)$ & $370(74,74 \%)$ & $362(54,19 \%)$ \\
\hline \multirow{4}{*}{ Origem* } & Autógena & $3(75 \%)$ & $19(23,17 \%)$ & $129(26,06 \%)$ & $189(28,29 \%)$ \\
\hline & Alógena & $1(25 \%)$ & $6(7,31 \%)$ & $22(4,44 \%)$ & $33(4,94 \%)$ \\
\hline & Xenógena & $0(0 \%)$ & $45(54,87 \%)$ & $212(42,82 \%)$ & $149(22,30 \%)$ \\
\hline & Aloplástica & $0(0 \%)$ & $25(30,48 \%)$ & $133(26,86 \%)$ & $401(60,02 \%)$ \\
\hline \multirow{4}{*}{ Composição* } & Cerâmica & $1(25 \%)$ & $16(19,51 \%)$ & $60(12,12 \%)$ & $156(23,35 \%)$ \\
\hline & Biovidro & $0(0 \%)$ & $1(1,21 \%)$ & $28(5,65 \%)$ & $22(3,29 \%)$ \\
\hline & Polímero & $0(0 \%)$ & $38(46,34 \%)$ & $177(35,75 \%)$ & $210(31,43 \%)$ \\
\hline & Compósito & $3(75 \%)$ & $27(32,92 \%)$ & $323(65,25 \%)$ & $407(60,92 \%)$ \\
\hline
\end{tabular}

Fonte: Autores, com base em dados de SBPqO (2020). *1 ou mais itens por categoria.

A Tabela 3 demonstra que a região Sudeste liderou pesquisas e fomentos ao longo de todas as décadas analisadas, as regiões Sul, Nordeste e Centro-Oeste cresceram significativamente na temática e a região Norte foi ausente em produção temática.

Tabela 3 - Frequência de pesquisas sobre biomateriais para ROG nas últimas quatro décadas conforme região e financiamento.

\begin{tabular}{lcccc}
\multicolumn{1}{c}{ Região } & $\mathbf{4 9 8 0}$ & $\mathbf{1 9 9 0}$ & $\mathbf{2 0 0 0}$ & $\mathbf{2 0 1 0}$ \\
Norte & $0(0 \%)$ & $0(0 \%)$ & $0(0 \%)$ & $0(0 \%)$ \\
Financiamento & $0(0 \%)$ & $0(0 \%)$ & $0(0 \%)$ & $0(0 \%)$ \\
Nordeste & $0(0 \%)$ & $1(1,22 \%)$ & $28(5,65 \%)$ & $67(9,20 \%)$ \\
Financiamento & $0(0 \%)$ & $0(0 \%)$ & $1(0,20 \%)$ & $11(1,51 \%)$ \\
Centro-Oeste & $0(0 \%)$ & $0(0 \%)$ & $2(0,40 \%)$ & $11(1,51 \%)$ \\
Financiamento & $0(0 \%)$ & $0(0 \%)$ & $0(0 \%)$ & $1(0,14 \%)$ \\
Sudeste & $4(100 \%)$ & $80(97,56 \%)$ & $421(84,88 \%)$ & $523(71,84 \%)$ \\
Financiamento & $0(0 \%)$ & $27(32,93 \%)$ & $158(31,85 \%)$ & $237(32,55 \%)$ \\
Sul & $0(0 \%)$ & $1(1,22 \%)$ & $45(9,07 \%)$ & $127(17,45 \%)$ \\
Financiamento & $0(0 \%)$ & $1(1,22 \%)$ & $3(0,60 \%)$ & $17(2,34 \%)$ \\
\hline
\end{tabular}

Fonte: Autores, com base em dados de SBPqO (2020). 


\section{Discussão}

A literatura demonstra alta variabilidade dos tipos de estudos apresentados na $\mathrm{SBPqO}$, onde pesquisas in situ oscilaram de 1\% (Sampaio et al., 2006) a 5\% entre as décadas de 2000 e 2010 (Palmeira et al., 2019), enquanto o total de pesquisas laboratoriais na década de 2010 perfizeram metade dos resumos (Xavier et al., 2011). Testes in vitro alcançaram 60\% do total na década de 1990 (Leles et al., 2006) e variaram nas décadas de 2000 a 2010 de 24\% (Morosini et al., 2012), 31\% (Dias et al., 2008), 43\% (Palmeira et al., 2019), 63\% (Barbosa et al., 2012), 64\% (Pontes et al., 2017) a 76\% (Sampaio et al., 2006). As pesquisas in vivo ocuparam 13\% dos resumos na década de 1990 (Leles et al., 2006), já entre as décadas de 2000 e 2010, 22\% (Fernandes Neto et al., 2019), 23\% (Sampaio et al., 2006), 24\% (Dias et al., 2008), 33\% (Barbosa et al., 2012) ou $38 \%$ só com animais (Palmeira et al., 2019) ou 38\% conjugadas a dentes humanos (Xavier et al., 2011). O rato foi o modelo animal mais usado para propostas de testagem craniana ou maxilo-mandibular (Barbosa et al., 2012; Cavalcanti et al., 2009), seguido do coelho (Cavalcanti et al., 2009), sendo o cão o mais raro (Barbosa et al., 2012). A justificativa para o camundongo não ser a primeira opção experimental residiria em sua melhor aplicabilidade a testes ectópicos de biocompatibilidade de biomateriais em tecido subcutâneo, etapa preliminar às análises ortotópicas em tecido ósseo, onde animais maiores são ideais (Sena et al., 2014; Jardelino et al., 2012; Lima et al., 2011; Almeida et al., 2010). É interessante notar que pesquisas que associaram as caracterizações in vitro e in vivo foram mais raras, na ordem de 3\% entre as décadas de 2000 a 2010 (Barbosa et al., 2012). Estudos clínicos apresentados na SBPqO alcançaram 12\% na década de 1990 (Leles et al., 2006), enquanto entre as décadas de 2000 e 2010 os valores foram de 3\% (Morosini et al., 2012), 28\% (Barbosa et al., 2012), 30\% (Dias et al., 2008) ou 74\% (Fernandes Neto et al., 2019), com menos de 1\% de estudos clínicos randomizados (Almeida-Marques et al., 2012).

Tais achados destoam do presente estudo, com maior expressividade in vivo seguida da clínica. A menor quantidade de pesquisas in vitro reflete a necessidade de maior busca por métodos alternativos ao uso de animais para testes de biomateriais, por outro lado, sem desconsiderar suas limitações técnicas e de eficácia (Cosme et al., 2017). De fato, as pesquisas normalizadas pré-clínicas por novos produtos odontológicos são fundamentais para progressão aos testes em humanos (Luz et al., 2020; Bittencourt et al., 2014; Lomelino et al., 2011).

As publicações brasileiras sobre aplicações de biomateriais para ROG em Odontologia têm demonstrado grande popularização de uso de enxertos ósseos na prática clínica (Araújo et al., 2020; Castro-Silva \& Coutinho, 2012). O controle da taxa de biodegradabilidade também têm despertado profundo interesse nesta temática, através de processamentos que incluem a oxidação para acelerar a biodegradação de materiais compactos (Luz et al., 2020), crosslinking para reforço estrutural de polímeros (Santana et al., 2009) ou tratamentos térmicos para cerâmicas (Lomelino et al., 2011; Silva et al., 2009), o que pode implicar desde a geração de esponjas hemostáticas com permanência de poucas semanas após implantação cirúrgica (Santana et al., 2009) a membranas e enxertos reabsorvíveis após meses de implantação, de forma equilibrada com a ROG esperada (Luz et al., 2020). O maior número de resumos da SBPqO também converge com o incremento expressivo nas últimas duas décadas de publicações sobre fibrina rica em plaquetas (PRF), um hemoderivado rico em fatores de crescimento angiogênicos e osteogênicos que tem se mostrado benéfica em ROG associada a biomateriais xenógenos ou aloplásticos (Castro-Silva, 2018). A versatilidade de uso, na forma de membrana, plug ou sticky bone, pode explicar o interesse crescente em PRF para preenchimento de alvéolos frescos e favorecimento da regeneração óssea (Ponte et al., 2019). O crescimento de pesquisas com aplicações para ROG poderia também estar associado à participação de especialidades que usam biomateriais em sua rotina clínica, como detectado nos resumos da SBPqO nas décadas de 1990 e 2000, com a área de periodontia presente acima de 10\% dos resumos (Fernandes Neto et al., 2019; Aquino et al., 2009; Dias et al., 2008; Leles et al., 2006; Cavalcanti et al., 2004), seguida da cirurgia bucomaxilofacial (Fernandes Neto et al., 2019; Cavalcanti et al., 2009; Leles et al., 2006) e da implantodontia (Aquino et al., 2009). 
A procedência xenógena de enxertos ósseos na Odontologia brasileira se mostrou evidente ao longo das décadas de 2000 (Castro-Silva et al., 2009), 2010 (Castro-Silva et al., 2013; Castro-Silva \& Coutinho, 2012) e 2020, junto a autógena (Araújo et al., 2020). Apesar do uso de aloenxertos ter quadruplicado em um quadriênio na região Sudeste (Castro-Silva et al., 2013), esta não constitui a preferência dos profissionais, o que pode ser um fator determinante na opção clínica da origem do biomaterial (Araújo et al., 2020; Castro-Silva \& Coutinho, 2012). O uso de produtos aloplásticos tem demonstrado um crescimento gradativo ao longo dos anos, o que pode estar associado à sua ampla disponibilidade e ao intenso desenvolvimento biotecnológico (Castro-Silva et al., 2021; Araújo et al., 2020; Castro-Silva et al., 2013; Castro-Silva \& Coutinho, 2012). A escolha de biomateriais baseada na eficácia biológica inclui biocompatibilidade, osteocondutividade de enxertos xenógenos frente autógenos (Bittencourt et al., 2014; Paulo et al., 2011; Castro-Silva et al., 2009) ou alógenos (Sousa et al., 2013) e dos promissores aloplásticos (Lomelino et al., 2011), ou estabilidade funcional de membranas osteopromotoras, naturais (Luz et al., 2020; Lima et al., 2010) ou sintéticas (Jardelino et al., 2012). Os presentes resultados corroboraram em parte a antiga dominância xenógena encontrada na literatura, lançando um olhar sobre a tendência atual de desenvolvimento de produtos aloplásticos para ROG.

Os enxertos ósseos cerâmicos constituem o padrão clássico de biomateriais para ROG, com alta aceitação pelos profissionais da Odontologia, vindo em sequência polímeros, compósitos e biovidros (Araújo et al., 2020; Castro-Silva \& Coutinho, 2012). Esse perfil reside nas desejáveis características físico-químicas de bioatividade e biomimetismo das biocerâmicas (Lomelino et al., 2011) e carreamento de fatores de crescimento ou células mesenquimais por polímeros a base de colágeno, poliácido lático e compósitos baseados em fosfatos de cálcio (Castro-Silva et al., 2010). Compósitos de hidroxiapatita e colágeno têm despertado muito interesse, pois a associação de alta carga mineral e matriz aglutinante favorece a reconstrução da microarquitetura e funcionalidade do tecido ósseo nativo (Gonçalves et al., 2009; Silva et al., 2009). Bow et al. (2019) levantaram a questão do impacto do processamento na funcionalidade de enxertos ósseos comerciais e verificaram que tanto a eficácia na regeneração óssea quanto uma boa relação custo-benefício são determinantes para o desenvolvimento de biomateriais osteobiológicos naturais ou sintéticos. Ainda, concepções de sustentabilidade e economia circular podem estimular o investimento biotecnológico em recursos naturais como fontes de novas matérias-primas, potencializando a criação de nanomateriais por síntese verde (Ribeiro et al., 2020) ou de compósitos e polímeros derivados de subprodutos agroindustriais (Castro-Silva et al., 2018), o que pode justificar a expressividade destas duas classes nos resumos analisados.

As pesquisas gerais da $\mathrm{SBPqO}$ se concentraram na região Sudeste, em particular no Estado de São Paulo, com médias próximas à metade do total da produção nacional, com flutuações nas posições seguintes entre as regiões Sul, Nordeste, Centro-Oeste e Norte, na década de 2000 (Fernandes Neto et al., 2019; Almeida-Marques et al., 2012; Barbosa et al., 2012; Lúcio et al., 2012; Morosini et al., 2012; Bezerra et al., 2011; Scariot et al., 2011; Aquino et al., 2009; Cavalcanti et al., 2009; Sampaio et al., 2006; Cavalcanti et al., 2004) e década de 2010 (Fernandes Neto et al., 2019; Gabardo et al., 2019; Perini et al., 2019; Silva \& Padilha, 2018; Pontes et al., 2017; Almeida-Marques et al., 2012; Barbosa et al., 2012; Lúcio et al., 2012; Bezerra et al., 2011).

Hipóteses para este comportamento residiriam nos achados de Castro-Silva et al. (2013), onde recursos humanos que trabalham com enxertos ósseos recuperados pela Plataforma Brasil, incluindo pesquisadores, profissionais e estudantes, se concentraram na região Sudeste, bem como de Cavalcante et al. (2008), que encontraram maior aglomeração de pesquisadores de produtividade do CNPq na área de Odontologia no Estado de São Paulo. Ainda, Dutra et al. (2012) observaram que abstracts brasileiros na IADR derivaram em massa de instituições da região Sudeste e do Estado de São Paulo, com maior cooperação científica com pesquisadores dos EUA na temática de materiais dentários. A produtividade nacional dos resumos da SBPqO na área de materiais dentários da década de 2000, na faixa de 13 a 23\% do total (Scariot et al., 2011), chegou a 27\% na década de 2010 (Gabardo et al., 2019), com relato isolado de 23\% na região Nordeste (Xavier et al., 2011), valores bem 
acima dos encontrados no presente estudo. Portanto, é confirmatória a heterogeneidade territorial da pesquisa odontológica diante do alto nível de evidências da literatura, que embora tenha deixado de ser menos desigual ao longo das décadas, mantém a maioria dos aglomerados imutáveis, consequência de redes de pesquisa mais consolidadas no país.

Com relação ao investimento geral em pesquisas no Brasil, os resumos da SBPqO exibiram entre as décadas de 2000 e 2010 as médias de financiamento de 15\% (Sampaio et al., 2006), 18\% (Cavalcanti et al., 2004), 23\% (Aquino et al., 2009), 26\% (Cavalcanti et al., 2009), 27\% (Xavier et al., 2011), 32\% (Pontes et al., 2017), 43\% (Barbosa et al., 2012) ou 44\% (Fernandes Neto et al., 2019), havendo discrepâncias de financiamento em comparação às décadas de 1980 a 2000 para 63\% dos resumos em patologia oral (Pereira et al., 2012) e entre as décadas de 2000 e 2010 para 2\% dos resumos em microcefalia (Queiroz \& Soares, 2019) ou 62\% em cariologia (Palmeira et al., 2019). Tem sido evidente nas publicações com resumos da SBPqO o forte apoio governamental de CNPq, CAPES, FINEP e fundações estaduais de amparo à pesquisa, destacando FAPESP, FAPERJ e FAPEMIG (Queiroz \& Soares, 2019; Pontes et al., 2017; Barbosa et al., 2012; Pereira et al., 2012; Xavier et al., 2011; Aquino et al., 2009; Cavalcanti et al., 2009; Sampaio et al., 2006; Cavalcanti et al., 2004), com raras citações ao aporte direto de instituições de ensino às pesquisas apresentadas no evento, na ordem de $2 \%$ na região Nordeste (Xavier et al., 2011) a 3\% no país (Barbosa et al., 2012).

Apesar da aplicação de recursos em materiais dentários estar compatível com estas faixas, chegando a 19\% na área (Cavalcanti et al., 2004), estes valores ainda estão aquém do ideal para garantir a viabilização de pesquisas odontológicas no Brasil dentro do escopo de biomateriais para ROG. É curioso notar este baixo financiamento na geração de tecnologias terapêuticas em contraste com o aquecido mercado de compras de materiais odontológicos, onde biomateriais de ROG de fonte importada persistem como escolha primária de cirurgiões-dentistas no Brasil (Castro-Silva \& Coutinho, 2012) e em uma cidade média da região Nordeste (Araújo et al., 2020), com apenas um estudo em uma cidade grande na região Sudeste indicando a fonte nacional mais prevalente (Castro-Silva et al., 2013). Estes dados reforçam a concentração de investimentos na região Sudeste como observada no presente estudo, bem como podem representar um potencial de inovação de biomateriais para ROG a explorar, mediante adequados recursos orçamentários.

Leles et al. (2006) observaram que 15\% dos resumos de 1999 da SBPqO foram convertidos em artigos, sendo a maioria das publicações em periódicos no extrato A da CAPES. Bergoli et al. (2011) verificaram em amostra de dez porcento dos resumos da SBPqO de 2001 que apenas 26,5\% foram publicados completos em periódicos, em maioria nos extratos B1 e B2 e no tempo médio de 24,1 meses, devido a morosidade das revistas ou falta de incentivo a autores. Tais valores estão aquém da taxa mundial média de 30\% de resumos apresentados em conferências odontológicas serem publicados como artigos (Hua et al., 2016). Cavalcante et al. (2008) observaram que pesquisadores de produtividade do CNPq da área de materiais odontológicos representam a maioria deste grupo, conduzindo estudos publicados em periódicos A internacional ou B nacional, o que relativizaria a produtividade nacional nesta temática. Primo et al. (2010) verificaram em um bacharelado de Odontologia na região Sul que o número de pesquisas institucionais apresentadas nas reuniões da SBPqO sofreu um crescimento substancial no final da década de 1990, porém seguido de estagnação e declínio significativo a partir do final da década de 2000. A cultura da publicação precisa ser ressignificada desde a graduação, pois pesquisas originais equivalem a apenas um terço dos trabalhos de conclusão e perdem sua atratividade para relatos de casos e revisões (Costa et al., 2015).

O presente estudo bibliométrico considerou resumos da SBPqO, que embora bem acessíveis e prestigiados na comunidade odontológica, correspondem a uma forma de literatura cinzenta, com resultados que podem diferir do número de publicações científicas completas ou patentes geradas no Brasil, dentro do escopo de biomateriais para ROG. Porém, nenhum outro estudo nacional se propôs a analisar estes resumos na mesma longa extensão de tempo, o que o torna robusto e um referencial metodológico para pesquisas afins. Desse modo, desvela a pertinência sobre a discussão geral da produtividade acadêmica ao longo das décadas, gerando um alerta sobre a quantidade e qualidade da divulgação científica da Odontologia 
brasileira, que carece de maior visibilidade.

\section{Conclusão}

O presente estudo indicou diferenças temporo-espaciais de produtividade acadêmica em pesquisas odontológicas brasileiras com biomateriais para regeneração óssea guiada através de resumos da SBPqO das últimas quatro décadas. O estudo do escopo de biomateriais para a regeneração óssea guiada indica sua gradativa evolução técnico-científica e reforça sua relevância histórica para a pesquisa odontológica brasileira.

Uma sugestão para trabalhos futuros residiria na correlação destes dados bibliométricos com a gestão do conhecimento e inovação dentro do escopo de biomateriais para ROG no cenário nacional.

\section{Agradecimentos}

Ao Governo do Estado do Ceará, à Fundação Cearense de Apoio ao Desenvolvimento Científico e Tecnológico (FUNCAP, fomento aos projetos BP3-0139-00270.01.00/18 e BP4-0172-00222.01.07/20), à Coordenação de Aperfeiçoamento de Pessoal de Nível Superior (CAPES, bolsas do Programa de Demanda Social, código 001), ao Conselho Nacional de Desenvolvimento Científico e Tecnológico (CNPq, Bolsa de Iniciação Científica) e à Universidade Federal do Ceará do Campus Sobral (UFC Sobral, Programa Institucional de Bolsas de Iniciação Científica/PIBIC e Programa Bolsa de Iniciação Acadêmica/PBIA), pelo apoio financeiro. A toda a equipe de trabalho do Laboratório de Biomateriais do Programa de Pósgraduação em Biotecnologia (PPGB-UFC Sobral), Curso de Odontologia (UFC Sobral), Grupo de Estudos em Morfologia (GEM-UFC Sobral) e Núcleo de Epidemiologia e Pesquisa Interprofissional em Saúde Coletiva (NEPIS-UFC Sobral), pelo apoio na concepção, coleta e análise dos dados e escrita desta pesquisa.

\section{Referências}

Almeida, M. S., Castro-Silva, I. I., Freire, M. V. \& Granjeiro, J. M. (2010). Reparo periapical com retro-obturações de MTA e super EBA em cães. Revista Brasileira de Odontologia, 67 (2), 188-193. http://dx.doi.org/10.18363/rbo.v67n2.p.188.

Almeida-Marques, R. V. D., Beber, A. J., Cavalcanti, Y. W., Guaré, R. O. \& Santos, M. T. B. R. (2012). Evidência científica da pesquisa odontológica brasileira em pacientes com necessidades especiais. RPG Revista de Pós Graduação, 19 (3), 107-112. http://revodonto.bvsalud.org/pdf/rpg/v19n3/a04v19n3.pdf.

Amorim, K. P. C., Alves, M. S. C. F., Germano, R. M. \& Costa, I. C. C. (2007). A construção do saber em Odontologia: a produção científica de três periódicos brasileiros de 1990 a 2004. Interface - Comunicação, Saúde, Educação, 11 (21), 9-23. http://dx.doi.org/10.1590/S1414-32832007000100003.

Aquino, S. N., Martelli, D. R. B., Bonan, P. R. F., Laranjeira, A. L. \& Martelli Júnior, H. (2009). Produção científica odontológica e relação com agências de financiamento de pesquisa. Arquivos em Odontologia, 45 (3), 142-146. https://periodicos.ufmg.br/index.php/arquivosemodontologia/article/view/3508.

Araújo, L. K., Antunes, G. S., Melo, M. M. \& Castro-Silva, I. I. (2020). Brazilian dentists' perceptions of using bone grafts: an inland survey. Acta odontologica latinoamericana, 33 (3), 153-164. http://actaodontologicalat.com/vol-33-issue-3-december-2020/.

Barbosa, V. L. S. A., Nóbrega, D. R. M. \& Cavalcanti, A. L. (2012). Estudo bibliométrico de pesquisas realizadas com fitoterápicos na Odontologia. Revista Brasileira de Ciências da Saúde, 16 (2), 123-130. http://dx.doi.org/10.4034/RBCS.2012.16.02.02.

Bergoli, C. D., Amaral, M., Rosa, R. A. \& Ardenghi, T. M. (2011). Subsequent publication of abstracts presented at the Brazilian Society of Oral Research Meeting. International Journal of Dentistry, 10 (4), 200-203. https://periodicos.ufpe.br/revistas/dentistry/article/view/14121/16984.

Bezerra, I. S. Q., Stadler, A. F., Almeida, I., Morosini, C., Scariot, R., Lima, A. A. S. \& Fernandes, A. (2011). Produção científica brasileira em radiologia odontológica e imaginologia nos anos de 2008 a 2011. Revista da Associação Brasileira de Radiologia Odontológica, 12 (2), 79-86. https://www.researchgate.net/profile/Amanda_Finger_Stadler/publication/267810290_Producao_cientifica_brasileira_em_radiologia_odontologica_e_imagino logia_nos_anos_de_2008_a_2011/links/547f42c50cf2cf248d9b264d.pdf.

Bittencourt, R. C., Castro-Silva, I. I., Paulo, A. O., Tavares, D. S. \& Granjeiro, J. M. (2014). Avaliação pré-clínica de um material substituto ósseo xenógeno a base de hidroxiapatita/colágeno. Revista Odonto Ciência, 29 (1), 6-13. http://dx.doi.org/10.15448/1980-6523.2014.1.12506.

Bow, A., Anderson, D. E. \& Dhar, M. (2019). Commercially available bone graft substitutes: the impact of origin and processing on graft functionality. Drug Metabolism Reviews, 51 (4), 533-544. http://dx.doi.org/10.1080/03602532.2019.1671860. 
Calasans-Maia, M. D., Mourão, C. F. A. B., Resende, R. F. B., Nascimento, J. R. B., Rossi, A. M. \& Granjeiro, J. M. (2017). Avaliação clínica de compósito constituído por fosfato de cálcio nanoestruturado e biopolímero associado à células osteoprogenitoras como alternativa à biocerâmica para a uso na rede SUS na regeneração óssea. Comunicação em Ciências da Saúde, 28 (1), 79-84. http://www.escs.edu.br/revistaccs/index.php/comum icacaoemcienciasdasaude/article/view/123.

Câmara, K. M. C. \& Nunes, I. M. (2021). A gestão do conhecimento no setor público: uma análise bibliométrica. Research, Society and Development, 10 (1), e8410111386. http://dx.doi.org/10.33448/rsd-v10i1.11386.

Castro-Silva, I. I. (2018). Platelet-rich fibrin for bone tissue regeneration. Otorhinolaryngology-Head and Neck Surgery, 3 (2), 1. http://dx.doi.org/10.15761/OHNS.1000171.

Castro-Silva, I. I. \& Coutinho, L. A. C. R. (2012). Uso de enxertos ósseos na Odontologia: perfil de cirurgiões-dentistas de Niterói/RJ. Revista Brasileira de Odontologia, 69 (2), 154-158. http://revodonto.bvsalud.org/scielo.php?script=sci_arttext\&pid=S0034-72722012000200003.

Castro-Silva, I. I., Coutinho, L. A. C. R. \& Granjeiro, J. M. (2010). Revisão sistemática sobre o uso de células-tronco mesenquimais em terapias de perdas ósseas. Innovations Implant Journal: Biomaterials and Esthetics, 5 (3), 29-34. http://revodonto.bvsalud.org/pdf/iij/v5n3/a07v5n3.pdf.

Castro-Silva, I. I., Ferreira, F. V. \& Maciel, J. A. C. (2021). Pesquisas em biotecnologia no Brasil: uma correlação espacial entre índice-H e desenvolvimento social. Research, Society and Development, 10 (1), e29910111807. http://dx.doi.org/10.33448/rsd-v10i1.11807.

Castro-Silva, I. I., Guerrero, J. A. P., Gomes, M. J. P. \& Souza Filho, M. S. M. (2018). Biotechnological potential of by-products of the Brazilian animal protein industry in the generation of xenogeneic biomaterials for bone regeneration. Trends in Research, 1 (3), 1-2. http://dx.doi.org/10.15761/TR.1000118.

Castro-Silva, I. I., Lima, F. M. S. \& Granjeiro, J. M. (2013). Enxertos ósseos na Odontologia brasileira: cenário, desafios e perspectivas na visão da Gestão em Saúde. Revista Fluminense de Odontologia, 1 (39), 63-71. http://dx.doi.org/10.22409/ijosd.v1i39.213.

Castro-Silva, I. I., Zambuzzi, W. F. \& Granjeiro, J. M. (2009a). Panorama atual do uso de xenoenxertos na prática odontológica. Innovations Implant Journal: Biomaterials and Esthetics, 4 (3), 70-75. https://www.researchgate.net/profile/Igor_Castro-Silva/publication/285839815_C urrent_overview_of_the_use_of_xenograft_in_dentistry/links/56d7149008aee1aa5f75c3c8/Current-overview-of-the-use-of-xenograft-in-dentistry.pdf.

Cavalcante, R. A., Barbosa, D. R., Bonan, P. R. F., Pires, M. B. O. \& Martelli-Júnior, H. (2008). Perfil dos pesquisadores da área de Odontologia no Conselho Nacional de Desenvolvimento Científico e Tecnológico (CNPq). Revista Brasileira de Epidemiologia, 11 (1), 106-113. http://dx.doi.org/10.1590/S1415$790 \times 2008000100010$.

Cavalcanti, A. L., Lucena, R. N., Martins, V. M. \& Granville-Garcia, A. F. (2009). Caracterização da pesquisa odontológica experimental em animais. Revista Gaúcha de Odontologia, 57 (1), 93-98. http://www.revistargo.com.br/viewarticle.php?id=955\&layout=abstract.

Cavalcanti, A. L., Melo, T. R. N. B., Barroso, K. M. A., Souza, F. E. C., Maia, A. M. A. \& Silva, A. L. O. (2004). Perfil da pesquisa científica em Odontologia realizada no Brasil. Pesquisa Brasileira de Odontopediatria e Clínica Integrada, 4 (2), 99-104.

Cosme, B., Falagan-Lotsch, P., Ribeiro, M., Napoleão, K., Granjeiro, J. M. \& Moura-Neto, R. (2017). Are your results valid? Cellular authentication a need from the past, an emergency on the present. In Vitro Cellular \& Developmental Biology - Animal, 53 (5), 430-434. http://dx.doi.org/10.1007/s11626-0160124-z.

Costa, R. M., Vieira, R. S., Cavalcanti, A. N., Tunes, U. R., Ribeiro, E. D. P. \& Oliveira, V. M. B. (2015). Perfil da pesquisa acadêmica odontológica da EBMSP. Revista da ABENO, 15 (1), 70-76. https://revabeno.emnuvens.com.br/revabeno/article/view/145.

Dias, A. A., Narvai, P. C. \& Rêgo, D. M. (2008). Tendências da produção científica em odontologia no Brasil. Revista Panamericana de Salud Pública, 24 (1), 54-60. http://dx.doi.org/10.1590/S1020-49892008000700007.

Dutra, D. M., Freires, I. A., Neves, R. S., Lima, D. M. B. \& Padilha, W. W. N. (2012). Aspectos Institucionais e Metodológicos dos Resumos Brasileiros Publicados na $85^{\mathrm{a}}$ Reunião Anual Mundial da IADR - 2007. Revista Brasileira de Ciências da Saúde, 16 (4), 545-552. http://dx.doi.org/10.4034/RBCS.2012.16.04.08.

Entradas, M., Bauer, M. W., O’Muircheartaigh, C., Marcinkowski, F., Okamura, A., Pellegrini, G., Besley, J., Massarani, L., Russo, P., Dudo, A., Saracino, B., Silva, C., Kano, K., Amorim, L., Bucchi, M., Suerdem, A., Oyama, T. \& Li, Y. Y. (2020). Public communication by research institutes compared across countries and sciences: building capacity for engagement or competing for visibility? PLoS One, 15 (7), e0235191. http://dx.doi.org/10.1371/journal.pone.0235191.

Fernandes Neto, J. A., Palmeira, P. T. S. S., Lacerda-Santos, J. T., Batista, A. L. A., Simões, T. M. S., \& Catão, M. H. C. V. (2019). Brazilian scientific research about acupuncture in Dentistry: bibliometric analysis of the papers presented at SBPqO meetings. Journal of Health Sciences, 21 (4), https://doi.org/10.17921/2447-8938.2019v21n4p348-51.

Gabardo, M. C. L., Copelli, F. A., Tuzzi, A. L., Trentin, G., Lima, J., Tomazinho, F. S. F. \& Sousa, Y. T. C. S. (2019). Pesquisa científica em endodontia apresentada na reunião anual da sociedade brasileira de pesquisa odontológica: análise bibliométrica de 2010 a 2018. Revista da ABENO, 19 (3), 144-152. http://dx.doi.org/10.30979/rev.abeno.v19i3.878.

Gonçalves, F., Hohn, A., Granjeiro, J. M., Silva, I. I. C., Taga, R., Cestari, T. M., Zanetti, R. V. \& Zanetti, A. L. (2009). Regeneração óssea em Odontologia com a utilização do substituto ósseo composto xenogênico Gen Mix. Implant News, 6 (4), 373-379. https://www.researchgate.net/publication/285976134_Bone_regeneration_in_Dentistry_with_use_of_composed_bone_substitute_Gen_Mix.

Hua, F., Walsh, T., Glenny, A. M. \& Worthington, H. (2016). Thirty percent of abstracts presented at dental conferences are published in full: a systematic review. Journal of Clinical Epidemiology, 75, 16-28. http://dx.doi.org/10.1016/j.jclinepi.2016.01.029.

Jardelino, C., Castro-Silva, I. I., Machado, C. P. G., Rocha-Leão, M. H., Rossi, A. M., Santos, S. R. A. \& Granjeiro, J. M. (2012). Biocompatibility analysis of a novel reabsorbable alloplastic membrane composed of alginate-Capsul. Revista Gaúcha de Odontologia, 60 (4), 419-423. http://revodonto.bvsalud.org/scielo.php?script=sci_arttext\&pid=S1981-86372012000400001\&lng=en\&nrm=iso. 
Leles, C. R., Rocha, S. S., Simões, P. A. \& Compagnoni, M. A. (2006). Taxa de publicação, na forma de artigos completos, de resumos apresentados em evento científico de pesquisa odontológica. Revista de Odontologia da UNESP, 35 (3), 141-147. https://www.revodontolunesp.com.br/article/588017db7f8c9d0a098b4945/pdf/rou-35-3-141.pdf.

Lima, C. J., Silva, I. I. C., Barros, L. F. H., Granjeiro, J. M. \& Silva, M. H. P. (2011). Resposta do tecido subcutâneo de camundongos à implantação de um novo biovidro à base de óxido de nióbio. Matéria (Rio de Janeiro), 16 (1), 574-582. https://doi.org/10.1590/S1517-70762011000100004.

Lima, C. J., Takamori, E. R., Hermida, L. F., Lenharo, A., Castro-Silva, I. I. \& Granjeiro, J. M. (2010). Porcine peritoneum as source of biocompatible collagen in mice. Acta cirurgica brasileira, 25 (4), 332-336. http://dx.doi.org/10.1590/\$0102-86502010000400006.

Lomelino, R. O., Castro-Silva, I. I., Linhares, A. B. R., Alves, G. G., Santos, R. A. S., Gameiro, V. S., Rossi, A. M. \& Granjeiro, J. M. (2011). The association of human primary bone cells with biphasic calcium phosphate ( $\beta$ TCP/HA 70: 30) granules increases bone repair. Journal of Materials Science: Materials in Medicine, 23 (3), 781-788. http://dx.doi.org/10.1007/s10856-011-4530-1.

Lúcio, P. S. C., Bezerra, K. F. O., Nonaka, C. F. W. \& Godoy, G. P. (2012). Câncer oral: caracterização da produção científica em odontologia do Brasil nos últimos cinco anos. RFO UPF, 17 (3), 280-284. https://doi.org/10.5335/rfo.v17i3.2455.

Luz, E. P. C. G., Chagas, B. S., Almeida, N. T., Borges, M. F., Andrade, F. K., Muniz, C. R., Castro-Silva, I. I., Teixeira, E. H., Popat, K., Rosa, M. F. \& Vieira, R. S. (2020). Resorbable bacterial cellulose membranes with strontium release for guided bone regeneration. Materials Science and Engineering: C, 116, e111175. http://dx.doi.org/10.1016/j.msec.2020.111175.

Marin, F., Rebello, M., Mello, A. L. S. F. \& Finkler, M. (2016). Ética e bioética como temas de pesquisa em odontologia: uma análise bibliométrica dos trabalhos apresentados nas reuniões da SBPqO. Revista da ABENO, 16 (4), 51-60. https://revabeno.emnuvens.com.br/revabeno/article/view/339/262.

Medeiros, A. A. \& Vasconcelos, A. C. F. (2020). Vulnerabilidade socioambiental: análise de redes bibliométricas. Research, Society and Development, 9 (9), e454997451. http://dx.doi.org/10.33448/rsd-v9i9.7451.

Menezes, K. R. \& Novaes, M. R. C. G. (2018). Formação pedagógica de professores de graduação em Enfermagem: revisão integrativa da literatura. Comunicação em Ciências da Saúde, 29 (4), 243-254. http://www.escs.edu.br/revistaccs/index.php/comunicacaoemcienciasdasaude/article/view/336/238.

Menezes, S. S. C., Corrêa, C. G., Silva, R. C. G. \& Cruz, D. A. M. L. (2015). Raciocínio clínico no ensino de graduação em enfermagem: revisão de escopo. Revista da Escola de Enfermagem da USP, 49 (6), 1037-1044. http://dx.doi.org/10.1590/S0080-623420150000600021.

Morosini, I. A. C., Otto, W. B., Carneiro, V. L., Oliveira, L. L. R. V., Oliveira, D. C. \& Ferreira, F. M. (2012). Profile of Brazilian scientific research in pediatric dentistry based on the $26^{\text {th }}$ Annual Meeting of the SBPqO. Revista Odonto Ciência, 27 (2), 132-136. http://dx.doi.org/10.1590/S198065232012000200007 .

Nobrega, T. E., Leôncio, L. L., Leite, M. S., Pereira, A. C. \& Silva, M. A. D. (2020). O impacto das Diretrizes Curriculares Nacionais na pesquisa científica nos cursos de Odontologia do Brasil. Research, Society and Development, 9 (8), e85984804. http://dx.doi.org/10.33448/rsd-v9i8.4804.

Palmeira, P. T. S. S., Leal, P. M., Fernandes Neto, J. A. \& Catão, M. H. C. V. (2019). Terapia fotodinâmica aplicada a cariologia: uma análise bibliométrica dos trabalhos apresentados na última década nas reuniões do SBPqO. Archives of Health Investigation, 8 (10), 586-591. https://doi.org/10.21270/archi.v8i10.3819.

Paulo, A. O., Castro-Silva, I. I., Oliveira, D. F., Machado, M. E. L., Bonetti Filho, I. \& Granjeiro, J. M. (2011). Repair of critical-size defects with autogenous periosteum-derived cells combined with bovine anorganic apatite/collagen: an experimental study in rat calvaria. Brazilian Dental Journal, 22 (4), 322-328. http://dx.doi.org/10.1590/S0103-64402011000400011.

Pereira, A. S., Shitsuka, D. M., Parreira, F. J. \& Shitsika, R. (2018). Metodologia da pesquisa científica. Santa Maria: UAB/NTE/UFSM. Recuperado em 6 fevereiro, 2021, de https://repositorio.ufsm.br/bitstream/handle/1/15824/Lic_Computacao_Metodologia-Pesquisa-Cientifica.pdf?sequence=1.

Pereira, J. S., Carvalho, C. H. P., Lima, E. N. A., Souza, E. L., Freitas, R. A. \& Galvão, H. C. (2012). Analysis of scientific production in oral pathology: a descriptive study. Arquivo Brasileiro de Odontologia, 8 (2), 16-22. https://repositorio.ufrn.br/bitstream/123456789/ 21790/1/Analysis\%20of\%20scientific\%20production_2012.pdf.

Perini, T., Ribeirete, E., Franco, A., Gabardo, G., Maciel, J. V. B. \& Fernandes, A. (2019). Quantifying the scientific studies in forensic dentistry presented at meetings of the "Sociedade Brasileira de Pesquisa Odontológica" (SBPqO) between 2013 and 2018. Revista Brasileira de Odontologia Legal, 6 (2), 41-49. https://doi.org/10.21117/rbol.v6i2.257.

Ponte, J. S., Araújo, M. A. D., Araújo, L. K. \& Castro-Silva, I. I. (2019). Platelet-rich fibrin: a versatile purpose for alveolar ridge preservation. Dental Oral Biology and Craniofacial Research, 2 (3), 1-3. http://dx.doi.org/10.31487/j.DOBCR.2019.03.05.

Pontes, K. T., Silva, E. L., Macêdo Filho, R. A., Silva, D. R. \& Lima, F. J. (2017). Estudo bibliométrico da produção científica em endodontia. Archives of Health Investigation, 6 (9), 435-438. http://dx.doi.org/10.21270/archi.v6i9.2221.

Primo, B. T., Grazziotin-Soares, R., Bertuzzi, D., Claudy, M. P., Hernandez, P. A. G. \& Fontanella, V. R. C. (2010). Produção científica da ULBRA: análise do número e do delineamento das pesquisas publicadas nos suplementos da Brazilian Oral Research (SBPqO). Stomatos, 16 (31), 69-76. http://revodonto.bvsalud.org/pdf/sto/v16n31/a08v16n31.pdf.

Queiroz, V. K. P. \& Soares, D. M. (2019). Pesquisas odontológicas relacionadas com microcefalia: um estudo bibliométrico. Archives of Health Investigation, 8 (11), http://dx.doi.org/10.21270/archi.v8i11.4720.

Quevedo-Silva, F., Santos, E. B. A., Brandão, M. M. \& Vils, L. (2016). Estudo bibliométrico: orientações sobre sua aplicação. Revista Brasileira de Marketing, 15 (2), 246-262. http://dx.doi.org/10.5585/remark.v15i2.3274. 
Ribeiro, J. J. K, Porto, P. S. S., Pereira, R. D. \& Muniz, E. P. (2020). Síntese verde de nanomateriais: trabalhos mais citados e tendências de pesquisa. Research, Society and Development, 9 (1), e54911593. http://dx.doi.org/10.33448/rsd-v9i1.1593.

Sampaio, F. C., Nunes, F. H. C., Barbosa, D. N. \& Cavalcanti, A. L. (2006). Análise das pesquisas em odontopediatria: estudo descritivo. Publicatio UEPG:

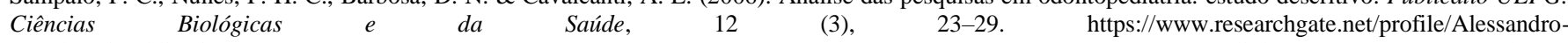
Cavalcanti/publication/277991238_ANALISE_DAS_PESQUISAS_EM_ODONTOPEDIATRIA_ESTUDO_DESCRITIVO/links/558c6e3208ae591c19da020 c/ANALISE-DAS-PESQUISAS-EM-ODONTOPEDIATRIA-ESTUDO-DESCRITIVO.pdf.

Santana, R. B., Silva, I. I. C., Gonçalves, F. L. L., Godinho, A. P. A. \& Mattos, C. M. L. (2009). Análise da resposta cicatricial da esponja Hemospon implantada em tecido conjuntivo de rato. Revista Brasileira de Implantodontia, 15 (4), 15-18. http://www.eurotechnew.pt/index_htm_files/2009\%20\%20RBI\%20-\%20Analise\%20da\%20resposta\%20cicatricial\%20-\%20Ronaldo\%20Barcellos.pdf.

SBPqO (2020). Publicações - Resumos. https://www.sbpqo.org.br/resumos/index.asp.

Scariot, R., Stadler, A. F., Assunção, C. M., Pintarelli, T. P. \& Ferreira, F. M. (2011). A map of Brazilian dental research in the last decade. Brazilian Oral Research, 25 (3), 197-204. http://dx.doi.org/10.1590/S1806-83242011000300002.

Sena, L. A., Almeida, M. S., Fernandes, G. V. O., Bretaña, R. M. G., Castro-Silva, I. I., Granjeiro, J. M. \& Achete, C. A. (2014). Biocompatibility of wollastonite-poly(N -butyl-2-cyanoacrylate) composites. Journal of Biomedical Materials Research Part B Applied Biomaterials, 102 (6), 1121-1129. http://dx.doi.org/10.1002/jbm.b.33093.

Sígolo, B. O. O. \& Silva, H. C. (2011). Destaque da produção científica brasileira em Odontologia no cenário mundial e a influência no comportamento informacional do profissional cirurgião-dentista $\quad$ (CD). Revista $\quad$ EDICIC, 189 -407. https://repositorio.unesp.br/bitstream/handle/11449/115301/ISSN2236-5753-2011-01-04-389-407.pdf?sequence=1\&isAllowed=y.

Silva, A. L. Ó. \& Padilha, W. W. N. (2018). Abordagens da produção científica odontológica brasileira sobre os efeitos da má oclusão e seus tratamentos na qualidade de vida. Revista Brasileira de Ciências da Saúde, 22 (4), 299-306. http://dx.doi.org/10.4034/RBCS.2018.22.04.02.

Silva, I. I. C., Pimentel-Soares, S., Bittencourt, R. C. \& Granjeiro, J. M. (2009). Natural bovine anorganic apatite and collagen presents osteoconductivity and contribute to bone repair of rat calvaria critical size defect. Key Engineering Materials, 396, 249-252. https://doi.org/10.4028/www.scientific.net/KEM.396398.249.

Sousa, S. B., Castro-Silva, I. I., Coutinho, L. A. C. R., Lenharo, A. \& Granjeiro, J. M. (2013). Osteoconduction and bioresorption of bone allograft versus anorganic bovine bone xenograft: a histomorphometric study in humans. Journal of Biomimetics, Biomaterials, and Tissue Engineering, 18, 85-95. https://doi.org/10.4028/www.scientific.net/JBBTE.18.85.

Xavier, A. F. C., Silva, A. L. O. \& Cavalcanti, A. L. (2011). Análise da produção científica em Odontologia no nordeste brasileiro com base em um congresso odontológico. Arquivos em Odontologia, 47 (3), 127-134. http://revodonto.bvsalud.org/pdf/aodo/v47n3/a03v47n3.pdf. 